Please note: This paper contains visible codes for electronic publishing. These will not appear in the final version and alignment of tables will be improved.

- Please check that all queries to author [in bold type and square brackets] have been answered, including those in the references and tables. Please do not renumber the references. Any additional references can be added to the bottom of the list and unwanted references deleted from the list without renumbering. The numbering will be corrected automatically during processing

$2320 ; 2292$

Christmas 2020:

\title{
Inspired by chance: valuing patients' informal contributions to research
}

Sebastian Crutch, ${ }^{1}$ professor of neuropsychology, Daniel Herron, ${ }^{2}$ head of research innovation, James Pickett, ${ }^{3}$ head of research, Simon Rosser, ${ }^{4}$ solicitor, Martin Rossor, ${ }^{1,2}$ national director for dementia research, on behalf of the Created Out of Mind team

${ }^{1}$ Dementia Research Centre, UCL Queen Square Institute of Neurology, University College London, London, UK

2 NIHR UCLH Biomedical Research Centre, London, UK

${ }^{3}$ Alzheimer's Society, London, UK

${ }^{4}$ Rare Dementia Support, London, UK

Correspondence to: S Crutch s.crutch@ucl.ac.uk

Serendipitous contributions from patients that influence the research agenda should be better recognised and acknowledged, argue Sebastian Crutch and colleagues

Public involvement is increasingly considered a required part of health research.

Typically defined as research being carried out "with" or "by" members of the public rather than "to," "about," or "for" them, ${ }^{1}$ public involvement is different from engagement (provision and dissemination of information and knowledge about research) and participation (taking part in a research study). Typical activities include identifying research priorities, ${ }^{2}$ choosing and co-designing outcome measures, ${ }^{3}$ advisory group membership, reviewing research materials, and carrying out the research.

Several major journals and funders have adopted strategies for enabling patients and the public to shape their aims and ways of working. Some also require researchers to make explicit statements about how - and importantly when - that involvement in the research process has occurred (eg, The BMJ's patient and public partnership campaign ${ }^{4}$ ). Other recent steps to improve standard practice include guidelines for researchers and the public, ${ }^{56}$ the development of public involvement standards, ${ }^{7}$ and suggested reporting guidelines (GRIPP2) for public involvement in research in research. ${ }^{8}$ These changes sit within a broader landscape of calls for greater involvement of patients and the public in scientific conferences, ${ }^{9}$ and 
autonomous patient-led movements to lead and crowdfund research ${ }^{1011}$ and innovate systems of care and support. ${ }^{12}$

A plethora of involvement frameworks have been developed. ${ }^{13}$ However, formal frameworks may not always capture the richness and variety of informal contributions to research that occur serendipitously, retrospectively, or independently. These informal contributions may originate in everyday conversations and interactions between researchers and people living with health conditions, including those with cognitive impairments that may make formal meetings and documents less appropriate.

\section{Inspiring new research and challenging assumptions}

When people with lived experience of dementia share their questions, experiences, and uncertainties, it may not be with any express intent to contribute to research. But these insights often have the effect of inspiring research by generating new ideas or hypotheses in the mind of the listener (or someone else with whom the gist of the conversation is later shared), challenging existing assumptions about their condition, or posing questions that can be investigated through academic research. Such conversations may take place during clinical interviews, at support group meetings, by letter or email, or over a cup of tea between research assessments. Critically, these inspiring contributions may occur at any time, not just conveniently during the planning phase of a project, and they might lead to new research activity immediately or some time later. One of the most striking examples of this we have known led to the discovery of the first gene for Alzheimer's (box 1).

\section{Box 1: Family connections}

Carol Jennings' father was diagnosed with Alzheimer's disease in his mid-50s. Subsequently an aunt and uncle began to experience symptoms, and she recalled her grandfather having similar problems. Describing events in 2010, Carol said: "I put two and two together and thought there's something funny going on here. This is the same family, developing the same symptoms, at a similar age ... I made a family tree, I found out lots of names and I thought this was interesting to someone who could do something about it."

Carol sent this information to MR's team and had an important role in the ensuing research, gathering together her whole family on the paternal side for blood tests. This research led to the discovery of the first gene for Alzheimer's disease. ${ }^{14}$

Open ended conversations with people with lived experience of a condition can also provide an opportunity to reflect on and question the status quo in a given area, and to challenge assumptions and standard practice. At a posterior cortical atrophy (PCA) support group meeting in London in March 2009, one member (co-author Simon Rosser) raised a simple but critical point that the use of different terminology for the condition (PCA, visual variant Alzheimer's disease, biparietal Alzheimer's disease, Benson's syndrome) resulted in a 
lack of understanding among patients, carers, and professionals. His comment sparked the formation of an international working party and the first multicentre research diagnostic criteria for PCA. ${ }^{15}$

The sharing of inspirational experiences is not limited to the spoken or written word, as we discovered when we had the privilege of working with William Utermohlen, a renowned artist primarily of portraits and murals. In 1995, he was diagnosed with Alzheimer's disease, and over the following five years created a series of self-portraits that altered strikingly in their style and representation of perceptual form and spatial relationship (fig 1). ${ }^{16}$ His work continues to communicate something about both the experience of living with a dementia and the biology of Alzheimer's disease that is more powerful than any brain scan, blood test, or scientific graph.

Posthumously, his comments and descriptions, and the lasting effect he had on clinicians and researchers, inspired the Created Out of Mind residency (2016-18) at The Hub, Wellcome Collection, which aimed to explore, challenge, and shape perceptions and understanding of dementias through science and the creative arts. ${ }^{17}$

Fig 1 William Utermohlen, Self Portrait with Saw 1997, 35.5 x 35.5 cm, Boïcos Collection, Paris. Courtesy of Chris Boïcos Fine Arts, Paris. [Q to A 1996? We will need permission from the owner of the portrait to use this and a jpeg, tiff, or eps image of at least $300 \mathrm{dpi}]$ ).

\section{Context setting and training}

Another informal contribution to research made by people with lived experience of dementia is enabling others to understand more of the context, place, and manner in which they live, or support someone to live, with their condition. Sometimes this takes the form of permitting researchers to observe the person in different scenarios, be it at home, while participating in an arts activity, or during clinical assessment. On other occasions the sharing may be more direct — through testimonies, giving talks, participating in demonstrations, or making audio and video recordings.

During the Created Out of Mind dementia residency, team members benefited greatly from the opportunity to observe dementia and arts practices. Seeing the effects of improvisatory music on people with advanced dementias participating in the Music for Life programme run by Wigmore Hall directly shaped the team's research priorities and objectives (fig 2). As a result $360^{\circ}$ camera based observational and quantitative rating techniques were developed to capture aspects of social interaction and musical, emotional, and physical engagement. ${ }^{18}$ 
Fig 2 Participants in a Music for Life session. (Source: Wigmore Hall @ James Berry [Q to A Do you have permission to use this? We will need a high quality file ]).

\section{Project design and development}

Shaping research design and development by participating in focus groups and advisory panels is an established component of public involvement. ${ }^{19}$ In recent years, co-production, referring to service providers and users working together to reach a collective outcome, has increased, particularly in translational research. ${ }^{20}$ In some cases, even formal involvement in co-design is founded on a much broader set of experiences shared between the person with lived experience and the researcher. Testimonies of researchers ${ }^{21}$ suggest that important ideas shaping research often emerge when people with a lived experience naturally and informally adopt an investigative or reflective stance in response to the challenges they face, which sometimes involves applying their previous professional skills to a problem area.

Two proponents of this kind of involvement in the co-design process are Pam and Richard Southerden, who have brought their experience of working in the IT industry to bear on technologies to support people living with dementia since Pam's diagnosis with PCA in July 2015. Working with neuropsychologist Aida Suarez Gonzalez and developer Ashley Peacock, they have influenced the design of reading app ReadClear. ${ }^{22}$ They have also collaborated with computer scientist Nick Firth and social scientist Emma Harding to explore the potential utility of Amazon Echo voice controlled devices in the homes of people with dementia related visual impairment. ${ }^{23}$

\section{Extra benefits from formal participation}

Involvement is typically distinguished from the many contributions people with dementia and their supporters make through participation (eg, enrolling in research, providing supportive information relating to a participant, supporting the participation of another person by accompanying them on a research visit). However, the benefits of participation often exceed the scientific objectives of the study.

In some cases, participation leads, intentionally or unintentionally, to contributions to research which go beyond the data acquired. For example, during a routine verbal memory test conducted as part of a drug trial, one participant with PCA made a surprising observation. Presented with the first in a series of printed words to read and remember-SAND - she commented, 'I can see an 'S' and I can see a 'D' but I can't see the letters in the middle." Her sharing of this hitherto unconsidered difficulty led to the design of experimental tests of spaced and unspaced letter identification (fig 3), the discovery of excessive visual crowding 
(difficulty identifying objects in clutter) in $\mathrm{PCA},{ }^{24}$ and the design of reading aids for people with dementia related visual impairment.

Fig 3 Flanked letter identification tests for visual crowding. Participants are asked to identify a central target letter surrounded by flanking letters, symbols or numbers (adapted from Yong et $\mathrm{al}^{24}$ ). People with excessive visual crowding can perceive the target letter reliably only when the flankers are spaced out (bottom line)

Just as formal involvement has been found to benefit both researchers and volunteers, ${ }^{19} 25$ so informal contributions can be valuable to all parties concerned. After reading a draft of the current article, Simon Rosser, whose wife had PCA, put into words what she felt: "The things that you are talking about, patient participation in research, patient groups such as the support group, particularly where the condition is not capable of being cured or treated in any meaningful way, is that the patient feels they are making a contribution and that they are doing something. Turning a natural feeling of helplessness in these situations into one where the patient is helping and feels that they are doing so gives the patient an enormous psychological boost. The very fact that people are interested in their views and in them (not just as a doctor treating them) and are wanting to understand them and what they are going through means a great deal to them."

\section{Creating opportunities}

The examples of informal research contributions here all relate to the dementias but have equivalents in many other areas of healthcare. All the examples have had a big effect on the research programme, but they are serendipitous and not predictable. Various strategies can increase opportunity for informal input into research (box 2), but these should be regarded as complementary not alternative to more established formal involvement and the structures, processes, and definitions that support it.

\section{Box 2: Practical suggestions for gaining informal input}

- Maximise opportunities to meet people living with a health condition-eg, sit in on a clinic where people are describing their situation or attend care homes open days and support groups. Basic scientists may find taking part in local science festivals fruitful

- Learn about lived experiences online. There are many patient and carer forums, often run by the disease charities, where people share their experiences, coping strategies, and solutions that provide a trove of potential informal inputs

- Ask open questions when meeting people — eg, "What is it you don't' understand about your condition?" and follow the conversation from there

- Be open to listening to all aspects of experience. Expect the unexpected, not just those symptoms, experiences, and questions traditionally recognised as part of the condition Collectively the stories of informal as well as formal involvement constitute an appeal to enable people affected by health conditions to influence the research which ultimately may affect them. In a world where research can often feel sanitised, these stories also encourage 
researchers to recognise how subtle interactions can influence emerging ideas. As Arends and Thackara wrote:

"Science is hypothesis driven ... but the first step on this journey-namely, the generation of the hypothesis itself, is rarely discussed... This early and ill-described stage entails a 'methodology gap'. It is at this messy and amorphous juncture, where the creation of knowledge starts, that scientists must find their inspiration." 26

In our experience, that inspiration often emerges through the experiences, questions, and uncertainties of those with a lived experience of a health condition.

Competing interests: We have read and understood BMJ policy on declaration of interests and have no relevant interests to declare.

We are grateful for conversations about public involvement with many friends and colleagues and especially other members of the Created Out of Mind, Rare Dementia Support, and Dementia Research Centre teams, particularly those named in the article and Emilie Brotherhood, Emma Harding, Paul Camic, Hannah Zeilig, Julian West, Tony Woods, Stuart Jennings, and Patricia Utermohlen. This work was supported by the Wellcome Trust [200783]. This work was also supported by Economic and Social Research Council (ESRC)/National Institute for Health Research (NIHR) (ES/L001810/1 and ES/S010467/1) and Alzheimer's Society (AS-PG-14-022) grants to SC. The Dementia Research Centre is supported by Alzheimer's Research UK, Brain Research Trust, and Wolfson Foundation. Rare Dementia Support is supported by the National Brain Appeal. This work was also supported by the NIHR Queen Square Dementia Biomedical Research Unit and the NIHR UCLH Biomedical Research Centre.

Patient and public involvement: This article has been particularly shaped by members of Rare Dementia Support and patients and carers attending, participating in, and shaping research and services at the Dementia Research Centre over more than 25 years. It is co-authored by someone who has given informal contributions to research (SR).

Patient consent obtained.

Competing interests: We have read and understood BMJ policy on declaration of interests and have no interests to declare.

$<$ eref $>1$ INVOLVE. What is public involvement in research? https://www.invo.org.uk/find-out-more/what-is-public-involvement-in-research-2/</eref>

<eref>2 James Lind Alliance, Alzheimer's Society. Outcomes of the James Lind Alliance dementia priority setting partnership. 2013. http://www.jla.nihr.ac.uk/prioritysetting-partnerships/dementia/downloads/Outcomes-of-the-Dementia-PSP-report.pdf $</$ eref $>$

$<$ eref $>3$ Comet Initiative. The development of a dementia core outcome set for dementia care in the community. https://www.comet-initiative.org/studies/details/677. $</$ eref $>$

<eref>4 BMJ patient and public partnership campaign. https://www.bmj.com/campaign/patient-partnership </eref>

<eref>5 INVOLVE. How to involve people in research. https://www.invo.org.uk/findout-more/how-to-involve-people/</eref>

<eref>6 INVOLVE. How to get involved. https://www.invo.org.uk/find-outmore/getting-involved/ </eref> 
<eref>7 National Institute for Health Research. Public involvement standards development. https://sites.google.com/nihr.ac.uk/pi-standards/home </eref>

$<$ jrn $>8 \quad$ Staniszewska S, Brett J, Simera I, et al. GRIPP2 reporting checklists: tools to improve reporting of patient and public involvement in research. BMJ 2017;358:j3453.

PubMed doi:10.1136/bmj.j3453 </jrn>

<eref>9 Engelen L. Patients not included. BMJ Opinion, 16 Aug 2013., https://blogs.bmj.com/bmj/2013/08/16/lucien-engelen-patients-not-included/) </eref>

<eref>10 The \#WeAreNotWaiting Diabetes DIY Movement. Healthline 2014. https://www.healthline.com/health/diabetesmine/innovation/we-are-not-waiting\#1</eref >

<eref $>11$ NIH All of Us Research Program. Sharon Terry. https://allofus.nih.gov/about/who-we-are/sharon-terry-ma</eref $>$

<eref>12 Shift.Ms. A social network for people with multiple sclerosis. https://shift.ms/</eref $>$

$<$ jrn>13 Greenhalgh T, Hinton L, Finlay T, et al. Frameworks for supporting patient and public involvement in research: Systematic review and co-design pilot. Health Expect 2019;22:785-801. PubMed doi:10.1111/hex.12888</jrn>

$<$ jrn>14 Goate A, Chartier-Harlin MC, Mullan M, et al. Segregation of a missense mutation in the amyloid precursor protein gene with familial Alzheimer's disease. Nature 1991;349:704-6. PubMed doi:10.1038/349704a0 </jrn>

$<$ jrn>15 Crutch SJ, Schott JM, Rabinovici GD, et al; Alzheimer's Association ISTAART Atypical Alzheimer's Disease and Associated Syndromes Professional Interest Area. Consensus classification of posterior cortical atrophy. Alzheimers Dement 2017;13:870-84. PubMed doi:10.1016/j.jalz.2017.01.014</jrn>

$<$ jrn>16 Crutch SJ, Isaacs R, Rossor MN. Some workmen can blame their tools: artistic change in an individual with Alzheimer's disease. Lancet 2001;357:2129-33. PubMed doi:10.1016/S0140-6736(00)05187-4</jrn>

$<$ jrn>17 Brotherhood E, Ball P, Camic PM, et al. Preparatory planning framework for Created Out of Mind: Shaping perceptions of dementia through art and science. Wellcome Open Res 2017;2:108. PubMed doi:10.12688/wellcomeopenres.12773.1</jrn>

$<$ jrn>18 Clare A, Camic PM, Crutch SJ, West J, Harding E, Brotherhood E. Using music to develop a multisensory communicative environment for people with late stage dementia. Gerontologist 2020;60:1115-25. doi:10.1093/geront/gnz169. PubMed</jrn>

<eref>19 Alzheimer's Society. "We can make our research meaningful": The impact of the Alzheimer's Society research network. https://www.alzheimers.org.uk/research/ourresearch/patient-and-public-involvement $</$ eref $>$

<eref>20 INVOLVE. Co-production. https://www.involve.org.uk/resources/methods/coproduction $</$ eref $>$

<eref>21 Suarez-Gonzalez A. How PPI worked for me. Researcher blog, 15 Nov 2018. https://www.dementiaresearcher.nihr.ac.uk/guest-blog-how-ppi-worked-for-me/ (accessed 22 Jan 2020).</eref $>$

$<$ jrn>22 Suarez-Gonzalez A, Ocal D, Pavisic I, et al. ReadClear: an assistive reading tool for people living with posterior cortical atrophy. J Alzheimers Dis 2019;71:1285-95. doi:10.3233/JAD-190335. PubMed</jrn>

<other>23 Firth N, Harding E, Sullivan MP, Crutch SJ, Alexander SC. ESCAPE — Echo scraper and classifier of persons: a novel tool to facilitate using voice-controlled devices for research. arXiv 2017:1706.0617. [Preprint.] https://arxiv.org/abs/1706.06176</other> 
Item: BMJ-UK; Article ID: crus54859;

Article Type: Standard article; TOC Heading: Feature; DOI: 10.1136/bmj.m4478

$<$ jrn>24 Yong KX, Shakespeare TJ, Cash D, et al. Prominent effects and neural correlates of visual crowding in a neurodegenerative disease population. Brain 2014;137:3284-99. PubMed doi:10.1093/brain/awu293 </jrn>

$<$ jrn $>25$ Bethell J, Commisso E, Rostad HM, et al. Patient engagement in research related to dementia: A scoping review. Dementia (London) 2018;17:944-75. PubMed doi:10.1177/1471301218789292</jrn>

$<$ bok>26 Arends B, Thackara D. Experiment: conversations in art and science. Wellcome Trust, 2003.</bok> 